\title{
EXPANSION OF HII REGIONS IN DENSITY GRADIENTS
}

\author{
José Franco $^{1,2}$, Guillermo Tenorio-Tagle ${ }^{2}$, and Peter Bodenheimer ${ }^{3}$. \\ 1 Instituto de Astronomía-UNAM, México. \\ ${ }^{2}$ Max-Planck Institut für Astrophysik, FRG. \\ ${ }^{3}$ Lick Observatory, USA.
}

\begin{abstract}
The main features of HII regions expanding in spherical and disk-like clouds with density gradients are reviewed. The spherical cases assume power-law density stratifications, $r^{-w}$, and the disk-like cases include exponential, gaussian, and sech $^{2}$ distributions. For power-law profiles, there is a critical exponent, $w_{\text {crit }}=3 / 2$, above which the ionization front cannot be "trapped" and the cloud becomes fully ionized. For clouds with $w<3 / 2$, the radius of the ionized region grows as $t^{4 /(7-2 w)}$ and drives a shock front into the ambient neutral medium. For $w=w_{\text {crit }}=3 / 2$ the shock wave cannot detach from the ionization front and the two move together with a constant speed equal to about $2 c_{i}$, where $c_{i}$ is the sound speed in the ionized gas. For $w>3 / 2$ the expansion corresponds to the "champagne phase", and two regimes, fast and slow, are apparent: between $3 / 2<w \leq 3$, the slow regime, the inner region drives a weak shock moving with almost constant velocity through the cloud, and for $w>3$, the fast regime, the shock becomes strong and accelerates with time.

For the case of disk-like clouds, which are assumed cylindrically symmetric, the dimensions of the initial HII regions along each azimuthal angle, $\theta$, are described in terms of the Strömgren radius for the midplane density, $R_{0}$, and the disk scale height, $H$. For $y_{0}=R_{0} \sin (\theta) / H \leq \alpha$ (where $\alpha$ is a constant dependent on the assumed density distribution) the whole HII region is contained within the disk, and for $y_{0}>\alpha$ a conical section of the disk becomes totally ionized. The critical azimuthal angle above which the HII region becomes unbounded is defined by $\theta_{\text {crit }}=\sin ^{-1}\left(\alpha H / R_{0}\right)$. The expansion of initially unbounded HII regions (i.e. with $y_{0}>\alpha$ ) proceeds along the $z$-axis and, if the disk column density remains constant during the evolution, the ionization front eventually recedes from infinity to become trapped within the expanding disk. For clouds threaded by a B-field oriented parallel to the symmetry axis, as expected in magnetically dominated clouds, this effect can be very prominent. The expanding gas overtaken by the receding ionization front maintains its linear momentum after recombination and is transformed into a high-velocity neutral outflow. In the absence of magnetic fields, the trapping has only a short duration.
\end{abstract}

\section{INTRODUCTION.}

For constant photon fluxes and uniform ambient densities, the evolution of HII regions has well defined formation and expansion phases (e. g. Kahn and Dyson, 1965; Mathews and O'Dell 1969; Spitzer 1978; Tenorio-Tagle 1982; Yorke 1986; Osterbrock 1989) During the formation phase, the stellar radiation field creates a supersonic ionization front whose velocity drops down to twice the sound speed in the ionized medium in, approximately, a recombination time. At this time, the end of the formation phase, 
the ionized region reaches the initial Strömgren radius (Strömgren 1939), and the pressure difference across the ionization front drives a shock wave into the ambient neutral medium. The radius of the expanding HII region, then, grows as $t^{4 / 7}$ afterwards. Changes in the boundary and initial conditions can result in significant departures from this simple evolution, however. A logical alternative, given that HII regions are created in molecular clouds, is to relax the assumption of a constant ambient density.

If the ionization front encounters a strong negative density gradient and overruns it, then the expansion will enter into its "champagne" phase. Numerical models of HII region evolution under these conditions have provided many details of the expected flows (Tenorio-Tagle 1982; Yorke 1986). The first models assumed discontinuities between constant density molecular and intercloud phases. Molecular clouds, however, have complex morphologies and density distributions, and they are clumpy structures composed of a variety of high-density condensations. These high-density condensations, or cloud fragments, seem to be the actual sites of star formation, and the initial shape and early evolution of the resulting HII regions depend on the corresponding fragment density distributions. Thus, further and refined numerical models have included more appropriate density gradients (e. g. Bodenheimer et al. 1983; Tenorio-Tagle et al. 1986). Given the complex evolution of the ionized flows, however, approximate analytical solutions are certainly useful in understanding the details of the dynamical phenomena.

Recently, the dynamical evolution in spherical clouds with power-law density distributions (Franco et al. 1989a, hereafter Paper I) and in disk-like clouds with a variety of stratifications (Franco et al. 1989b, hereafter Paper II) has been derived analytically. The results of these works, aside from reinforcing the numerical modeling, provide a physical insight to the rich variety of phenomena that can appear during the HII evolution in decreasing density gradients. These include the generation of internal shocks in clouds with power-law density stratifications and, perhaps more remarkable, the existence of receding ionization fronts in disk-like regions. Here we review the main features of the evolution reported in these papers.

\section{HII REGIONS IN POWER-LAW DENSITY DISTRIBUTIONS}

Following Paper I, for a spherical cloud with a molecular density distribution (including a central core, with radius $r_{c}$ and constant density $n_{c}$, and an envelope with a power-law density stratification)

$$
n_{H_{2}}(r)= \begin{cases}n_{c} & \text { for } r \leq r_{c} \\ n_{c}\left(r / r_{c}\right)^{-w} & \text { for } r \geq r_{c}\end{cases}
$$

the size of the initial HII region is derived, as usual, by equating the total number of ionizing photons emitted by the star per unit time to the total number of recombinations per unit time within the ionized volume. For $w \neq 3 / 2$, this initial radius can be written as

$$
R_{w}=g(w) R_{s}
$$

with

$$
g(w)=\left[\frac{3-2 w}{3}+\frac{2 w}{3}\left(\frac{r_{c}}{R_{s}}\right)^{3}\right]^{\beta}\left(\frac{R_{s}}{r_{c}}\right)^{2 w \beta}
$$


where $R_{s}$ is the Strömgren radius for the density $n_{c}$. The solution for $w=3 / 2$ is

$$
R_{3 / 2}=r_{c} \exp \left\{\frac{1}{3}\left[\left(\frac{R_{s}}{r_{c}}\right)^{3}-1\right]\right\} .
$$

Equation (3) defines a critical exponent for the formation phase, $w_{f}$, above which the solution for $R_{w}$ does not exist

$$
w_{f}=\frac{3}{2}\left[1-\left(\frac{r_{c}}{R_{s}}\right)^{3}\right]^{-1},
$$

and corresponds to the maximum density gradient that is able to "trap" the ionization front (i.e. recombinations and new ionizations in steeper gradients are not sufficient to slow down the ionization front, which remains indefinitely as a weak R-type front). Note that for $R_{s} / r_{c}>2$ the critical value becomes $w_{f} \simeq 3 / 2$.

After the formation phase has been completed in clouds with $w \leq w_{f}$, the pressure in the ionized region drives a shock into the molecular ambient medium and the HII region begins its expansion phase. For simplicity, we asssume that the shock evolution starts at $t=0$ when $R_{w}$ is achieved. Given that the expansion is subsonic with respect to the ionized gas, the density structure inside the HII region can be regarded as uniform and its average ion density at time $t$ is

$$
\rho_{i}(t) \simeq \mu_{i} \frac{(9-6 w)^{1 / 2}}{3-w}\left(2 n_{c}\right)\left(R_{s} / R(t)\right)^{3 / 2},
$$

where $\mu_{i}$ is the mass per ion, and $R(t)$ is the radius of the HII region at the time $t$.

For $w \leq 3 / 2$, the radius can be approximated by

$$
R(t) \simeq R_{w}\left[1+\frac{7-2 w}{4}\left(\frac{12}{9-4 w}\right)^{1 / 2} \frac{c_{i} t}{R_{w}}\right]^{4 /(7-2 w)}
$$

where $c_{i}$ is the sound speed in the ionized gas. The ratio of total mass (neutral plus ionized), $M_{s}(t)$, to ionized mass, $M_{i}(t)$, contained within the expanded radius evolves as

$$
\frac{M_{s}(t)}{M_{i}(t)} \simeq\left[\frac{R(t)}{R_{w}}\right]^{(3-2 w) / 2}
$$

Equation (8) indicates: i) for $w<3 / 2$, the interphase between the ionization front and the leading shock accumulates neutral gas and its mass grows with time to exceed even the mass of ionized gas, and ii) for $w=3 / 2=w_{\text {crit }}$, the two fronts move together without allowing the formation and growth of a neutral interphase. Note that the decreasing ratio predicted by the equation for $w>3 / 2$ is physically meaningless and it only indicates that the ionization front overtakes the shock front (and proceeds to ionize the whole cloud). Thus, regardless of the value of the critical exponent for the formation phase, $w_{f}$, the expansion phase is characterized by a critical exponent with a well defined value, $w_{\text {crit }}=3 / 2$, which is independent of the initial conditions. Furthermore, this critical exponent $w_{\text {crit }}=3 / 2$ is not affected by dust absorption (Paper I).

For $3 / 2<w<w_{f}$, the ionization front eventually overtakes the shock and soon the whole cloud becomes ionized. At that moment the gas acquires the same temperature 
everywhere, and the pressure gradient simply follows the density gradient. All parts of the cloud are then set into motion, but the expanded core (now with a radius identical to the position of the overtaken shock) is the densest region and feels the strongest outwards acceleration. Thus, superimposed on the general gas expansion there is a wave driven by the fast growing core (the wave location defines the size of the expanded core), and the cloud experiences the so-called "champagne" phase. This core expansion tends to accelerate with time and two different regimes, separated by $w=3$, are apparent: a "slow" regime with almost constant expansion velocities, and a "fast" regime with strongly accelerating shocks.

The slow regime corresponds to $3 / 2<w<3$ and the core grows approximately as

$$
r(t) \simeq r_{c}+\left[1+\left(\frac{3}{3-w}\right)^{1 / 2}\right] c_{i} t
$$

where for simplicity the initial radius of the denser part of the cloud has been set equal to $r_{c}$, the initial size of the core. For $w=3$ the isothermal growth is approximated by

$$
r(t) \simeq 3.2 r_{c}\left[\frac{c_{i} t}{r_{c}}\right]^{1.1} .
$$

For $w>3$, the fast regime, the shock acceleration increases with increasing values of the exponent and the core expansion is approximated by

$$
r(t) \simeq r_{c}\left[1+\left(\frac{4}{w-3}\right)^{1 / 2}\left(\frac{\delta+2-w}{2}\right) \frac{c_{i} t}{r_{c}}\right]^{2 /(\delta+2-w)},
$$

where

$$
\delta \simeq 0.55(w-3)+2.8
$$

\section{HII REGIONS IN DISK-LIKE CLOUDS}

All stratifications likely to represent a "disk-like" cloud, or a gaseous disk, decrease faster than any power-law at large radii. Therefore, all these distributions have a critical point beyond which their density fall-off is steeper than $r^{-3 / 2}$, and can be completely ionized beyond that point. Following Paper II, the definition of a gaseous disk is simply an elongated cloud which can be regarded as cylindrically symmetric. The variables $r$, $\theta$, and $z$ are taken as the usual cylindrical coordinates. The gas is assumed molecular with a constant scale height, $H$, and the ionizing source is located at midplane. The density distribution along the $z$-axis is

$$
n(z)=n_{0} G(z / H),
$$

where $n_{0}$ is the density at midplane, and $G(z / H)$ is any of the functions $e^{-z / H}, e^{-z^{2} / H^{2}}$, and $\operatorname{sech}^{2}(z / H)$. Clearly, there is a critical point for each one of these cases.

The initial shape of the ionized region is defined by the equilibrium between photoionization and recombination along each solid angle. Defining the dimensionless variables $y=r \sin (\theta) / H$ and $y_{0}=R_{0} \sin (\theta) / H$ (where $R_{0}$ is the Strömgren radius for the midplane density), the shape is given by the integral equation 


$$
y_{0}=\left[3 \int_{0}^{y} y^{2} G^{2}(y) d y\right]^{1 / 3},
$$

and the resulting size of the initial HII region as a function of the angle $\theta$ can be written as

$$
R_{\theta} \simeq R_{0} h(\theta)
$$

with the "shape" function

$$
h(\theta)=\left(\frac{\alpha}{2 y_{0}}\right) \ln \left[\frac{1+\left(y_{0} / \alpha\right)}{1-\left(y_{0} / \alpha\right)}\right],
$$

where $\alpha=\left[3 \int_{0}^{\infty} y^{2} G^{2}(y) d y\right]^{1 / 3}$ and has the values $0.78,0.88$, and 0.91 for the gaussian, sech $^{2}$, and exponential cases, respectively. For small values of $y_{0}$ (i.e. $y_{0} \leq 0.3$ ), the shape function reduces to

$$
h(\theta) \simeq\left(1+\frac{y_{0}^{2}}{3 \alpha^{2}}\right) .
$$

The critical point is defined by $y_{0}=\alpha$, and this corresponds to a critical angle

$$
\theta_{\text {crit }}=\sin ^{-1}\left(\alpha \frac{H}{R_{0}}\right)
$$

which defines the conical section of the disk that is fully ionized. Note that the HII region reaches the critical point when $R_{0}>\alpha H$, but it becomes completely bounded within the disk when $R_{0} \leq \alpha H$.

The early stages of the expansion are controlled by the density gradient perpendicular to midplane (see Bodenheimer et al. 1983) but, as discussed in Paper II, the details of the general evolution are affected by lateral gas velocity components generated near the boundaries with the neutral gas. These two-dimensional effects cannot be treated with a simple analytical scheme. Note, however, that the presence of a $B$ field oriented in the direction of the disk symmetry axis, as expected in magnetically supported clouds, inhibits the lateral gas movements and the ionized region is forced to expand only along the $z$-axis (allows for a simple one-dimensional treatment of the expansion). For $B=0$, on the other hand, two dimensional effects become important after a sound crossing time, and this restricts the discussion in the nonmagnetic case to the early stages of the expansion.

The analysis of the magnetic and nonmagnetic cases is performed only along the symmetry axis $(\theta=\pi / 2)$. The symbols $n_{0}, R_{0}, H$, and $y_{0}$ are kept as the initial values (i.e. at $t=0$ ) of the corresponding parameters, and the discussion is restricted to initially unbounded regions $\left(y_{0}>\alpha\right)$.

a) Expansion with $B$-fields: high-velocity neutral outflows

The initial gas acceleration (defined by the density gradient) stretches the whole disk and the gas velocities can reach supersonic values. The magnetic field, oriented along the $z$-axis, is assumed uniform and the flow is constrained to move within the flux tubes. Thus, the evolution of the flow can be analysed in one dimension. A rough upper bound to the required field strength at midplane is $B \sim 10^{-4} n_{3}^{1 / 2} c_{1} \mathrm{G}$, where $n_{3}=n_{0} / 10^{3} \mathrm{~cm}^{-3}$ and $c_{1}=c_{i} / 10 \mathrm{~km} \mathrm{~s}^{-1}$. 
At early times, $t \leq 2 H / c_{i}$, the scale height grows as

$$
H(t) \simeq H\left(1+\beta c_{i}^{2} t^{2} / H^{2}\right),
$$

with $\beta$ equal to $1,0.76$, and 0.5 for the gaussian, sech $^{2}$, and exponential stratifications, respectively. Similarly, at these early times, the midplane density decreases as

$$
n_{0}(t) \simeq n_{0}\left(1+\beta c_{i}^{2} t^{2} / H^{2}\right)^{-1} .
$$

Later, for $t \geq 2 H / c_{i}$, the late times approximation is

$$
n_{0}(t) \simeq \frac{n_{0} H}{(2 \beta+0.5) c_{i} t},
$$

which is equivalent to define a late times scale height (enclosing an almost uniform density) $H(t) \sim(2 \beta+0.5) c_{i} t$.

The size of the HII region along midplane grows approximately as

$$
R_{0}(t) \simeq R_{0}\left[\frac{n_{0}}{n_{0}(t)}\right]^{2 / 3}
$$

Note that if $R_{0}(t)$ becomes smaller than $H(t)$, the HII region will be trapped within the expanded disk. The growth rate for $R_{0}(t)$ and $H(t)$ are different, and the dimensionless variable $y_{0}(t)=R_{0}(t) / H(t)$ is a decreasing function of time. At early times it evolves as

$$
y_{0}(t) \simeq y_{0}\left[1+\beta\left(\frac{c_{i} t}{H}\right)^{2}\right]^{-1 / 3},
$$

and at late times it is approximated by

$$
y_{0}(t) \simeq y_{0}\left[\frac{H}{(2 \beta+0.5) c_{i} t}\right]^{1 / 3} .
$$

Thus, there exists a "trapping" time, $\tau_{t}$, at which the value of $y_{0}(t)$ drops below unity and the expanding HII region becomes ionization bounded in the $z$-direction. At this time, which becomes longer with increasing values of $y_{0}$, the ionization front recedes down to a location inside $H(t)$ and the gas exterior to this point recombines.

For $y_{0} \leq 1.4$, the trapping occurs when the midplane density reaches the value

$$
n_{0}\left(\tau_{t}\right) \simeq\left(\alpha / y_{0}\right)^{3} n_{0}
$$

and the trapping time is defined by

$$
\tau_{t} \simeq \frac{H}{c_{i} \beta^{1 / 2}}\left[\left(\frac{y_{0}}{\alpha}\right)^{3}-1\right]^{1 / 2}
$$

For $y_{0} \geq 1.4$, the trapping time is approximated by

$$
\tau_{t} \simeq \frac{H y_{0}^{3}}{(2 \beta+0.5) c_{i}},
$$

when the corresponding midplane density is 


$$
n_{0}\left(\tau_{t}\right) \simeq\left(n_{0} / y_{0}^{3}\right)
$$

A lower bound to the mass of the recombining zone is

$$
M_{r e c} \sim \mu \pi R_{0}^{2}\left(\tau_{t}\right) n_{0} H \int_{1}^{\infty} G(x) d x \simeq 0.3 \mu \pi R_{0}^{2} y_{0}^{4} n_{0} H,
$$

where $\mu$ is the mass per particle. This gas maintains its momentum after recombination and becomes a neutral high-velocity outflow. Defining $R_{17}=R_{0} / 10^{17} \mathrm{~cm}, H_{17}=$ $H / 10^{17} \mathrm{~cm}, c_{1}=c_{i} / 10 \mathrm{~km} \mathrm{~s}^{-1}$, and $n_{6}=n_{0} / 10^{6} \mathrm{~cm}^{-3}$, the lower limits to the mass and momentum locked in the high-velocity neutral flow are $M_{\text {rec }} \sim 0.1 R_{17}^{2} n_{6} H_{17} y_{0}^{4} M_{\odot}$ and $P_{\text {rec }} \sim 2 R_{17}^{2} n_{6} H_{17} y_{0}^{4} c_{1} M_{\odot} \mathrm{km} \mathrm{s}^{-1}$. These approximate expressions indicate that magnetic cases with $y_{0}>1$ can generate powerful neutral outflows.

\section{b) Expansion with $B=0$ : short trapping stages}

In this case, the expansion is affected by lateral gas motions driven by rarefaction waves. These waves reach the symmetry axis in a sound crossing time, $\tau_{2 D} \simeq R_{0} / c_{i}$. Thus, the trapping occurs only at the early stages of expansion and its properties are given by equations (24) and (25). The trapping time can be written as

$$
\tau_{t} \simeq \frac{\tau_{2 D}}{y_{0} \beta^{1 / 2}}\left[\left(\frac{y_{0}}{\alpha}\right)^{3}-1\right]^{1 / 2}
$$

and given that $\tau_{t} \tau_{2 D}$, the existence of the trapping is restricted to cases $\alpha<y_{0} 1$. Also, the trapping is a short lived event and the ionization front moves again outwards after a time $\tau_{2 D}$.

\section{CONCLUSIONS}

The results summarized in this paper provide the main features, under a wide range of conditions, of the dynamical evolution of ionized regions in decreasing density gradients.

For spherical clouds with a power-law density distribution, $r^{-w}$, there is a critical exponent above which the cloud becomes completely ionized. Its value in the formation phase depends on the initial conditions, but it has a well-defined value, $w_{\text {crit }}=3 / 2$, during the expansion phase. For $w \leq w_{\text {crit }}$, the radius evolves as $t^{4 /(7-2 w)}$. Cases with $w>w_{\text {crit }}$ follow the champagne phase, where the expansion becomes supersonic, and two well defined regimes can be separated. For $3 / 2<w \leq 3$, the slow regime, the core expansion is mildly supersonic and has almost constant velocity. For $w>3$, the fast regime, the core expansion drives a strongly accelerating shock. These results indicate, aside from the rich variety of dynamical phenomena, that optical HII regions are density bounded and that some star forming regions (those with $w$ larger than 3 ) may be likely sources for cosmic ray acceleration.

For disk-like clouds, with and without magnetic fields, the evolution also presents several interesting properties. Some details depend on the particular density profile, but the main features are determined by the scale height and the initial Strömgren radius along midplane. The initial shape of the ionized region has a critical point, $y_{0}=\alpha$, which defines a fully ionized conical section in the disk. For initially unbounded regions, $y_{0}>\alpha$, the evolution can produce large expansion velocities. Moreover, the expanded disk can trap the ionization front and the HII region becomes bounded. If a $B$-field oriented parallel to the $z$-axis is present, the event is long lasting and produces a 
powerful and supersonic neutral outflow. In the absence of magnetic fields, the lifetime of the trapping stage is limited by two-dimensional effects.

All these features certainly warrant further research. Future developments and the application of these results to particular objects will be explored in forthcoming works.

\section{ACKNOWLEDGMENTS}

P. B. and J. F. acknowledge the hospitality of the Max-Planck Institut für Astrophysik. J. F. acknowledges partial financial support from CONACyT-México. G. T.-T. is supported by the Deutsche Forschungsgemeinschaft grant Mo 416.

\section{REFERENCES}

Bodenheimer, P., Yorke, H. W., Tenorio-Tagle, G., and Beltrametti, M. 1983, in Supernova Remnants and Their X-Ray Emission, IAU Symp. 101, eds. J. Danziger and P. Gorenstein (Dordrecht: Reidel Publ. Co.), p. 399.

Franco, J., Tenorio-Tagle, G., and Bodenheimer, P. 1989a, Ap. J., in press (Paper I).

Franco, J., Tenorio-Tagle, G., and Bodenheimer, P. 1989b, Rev. Mex. Astron. Astrofís., in press (Paper II).

Kahn, F. D., and Dyson, J. E. 1965, Ann. Rev. Astron. Ap., 3, 47.

Mathews, W. G., and O'Dell, C. R. 1969, Ann. Rev. Astron. Ap., 7, 67.

Osterbrock, D. E. 1989, Astrophysics of Gaseous Nebulae and Active Galactic Nuclei (Mill Valley: University Science Books).

Spitzer, L. 1978, Physical Processes in the Interstellar Medium (New York: Wiley and Sons).

Strömgren, B. 1939, Ap. J., 89, 526.

Tenorio-Tagle, G. 1982, in Regions of Recent Star Formation, eds. R. S. Roger and P. E. Dewdney (Dordrecht: Reidel Publ. Co.), p. 1.

Tenorio-Tagle, G., Bodenheimer, P., Lin, D. N. C., and Noriega-Crespo, A. 1986, M. N. R. A. S., 221, 635.

Yorke, H. W. 1986, Ann. Rev. Astron. Ap., 24, 49. 